\title{
UNIQUE HAHN-BANACH EXTENSIONS AND SIMULTANEOUS EXTENSIONS
}

\author{
MORISUKE HASUMI
}

\begin{abstract}
ABSTRACr. In this note we mention certain connections existing between unique Hahn-Banach extensions and simultaneous extensions. We also describe an application of a continuous selection theorem to simultaneous extensions.
\end{abstract}

1. Effects of unique Hahn-Banach extensions. Let $(m)$ be the Banach space of bounded sequences of real (or complex) numbers with the supremum norm and $\left(c_{0}\right)$ the subspace of $(m)$ consisting of sequences converging to zero. Then the following holds, which is ascribed to H. P. Rosenthal (cf. Banilower [1, Proposition 1.2]): If $T$ is a linear operator from $(m)$ into a normed linear space $F$ with $\|T\|=1$ such that $T$ is an isometry on $\left(c_{0}\right)$, then $T$ is an isometry on ( $m)$. If, moreover, $F=(m)$ and $T$ is the identity operator on $\left(c_{0}\right)$, then $T$ is the identity operator on $(m)$. Our first objective is to describe principles underlying this result.

Let $E$ be a normed linear space with dual $E^{*}, L$ a subspace of $E, T$ a linear operator from $E$ into a normed linear space $F$ with $\|T\|=1$ such that $T$ is an isometry on $L$, and $M=T(L)$, which is considered as a subspace of $F$. These notations are preserved through this section. Let $S^{*}(E, L)$ be the set of $x^{*} \in E^{*}$ with $\left\|x^{*}\right\|=1$ such that $x^{*}\left|L=y^{*}\right| L$ implies $x^{*}=y^{*}$ if $\left\|y^{*}\right\|$ $\leqslant 1$. If $x^{*} \in S^{*}(E, L)$, then $\left\|x^{*} \mid L\right\|=1$ and $x^{*}$ is the unique Hahn-Banach extension of $x^{*} \mid L$ to $E$. We denote by $E^{*}(L)$ the subspace of $E^{*}$ generated by the $\sigma\left(E^{*}, E\right)$-closed convex hull of $S^{*}(E, L)$ and by $E^{*}(L)^{\perp}$ the orthogonal complement of $E^{*}(L)$ in the space $E$. By the Krein-Smulian theorem (cf. Dunford [3, V.5.8]), $E^{*}(L)$ is $\sigma\left(E^{*}, E\right)$-closed. For a given subset $D$ of $E$ we say that an element $x \in E$ is orthogonal to $D$ if $\left\|x+c x^{\prime}\right\| \geqslant\|x\|$ for any $x^{\prime} \in D$ and any scalar $c$. Consider the case in which $D$ is a closed subspace. Since $D^{\perp}$, the orthocomplement of $D$ in $E^{*}$, is regarded as the dual of the quotient space $E / D$, we have

$$
\|x\| \geqslant \inf \left\{\left\|x+x^{\prime}\right\|: x^{\prime} \in D\right\}=\sup \left\{\left|\left\langle x, x^{*}\right\rangle\right|: x^{*} \in D^{\perp} \text { and }\left\|x^{*}\right\|=1\right\} .
$$

Received by the editors February 6, 1976 and, in revised form, June 29, 1976 AMS (MOS) subject classifications (1970). Primary 46B99, 46E15.

Key words and phrases. Hahn-Banach extension, simultaneous extension, continuous selection. 
The equality sign prevails if and only if $x$ is orthogonal to $D$. As for the isometric property of $T$ we have the following

THEOREM 1. $\|T x\|=\|x\|$ for $x \in E$, if either $x \in L$ or $x$ is orthogonal to the subspace $E^{*}(L)^{\perp}$.

Proof. Take any $x^{*} \in S^{*}(E, L)$ and set $v^{*}=x^{*} \circ(T \mid L)^{-1}$. Then $v^{*}$ is a linear functional on the subspace $M=T(L)$ and $\left\|v^{*}\right\|=\left\|x^{*} \mid L\right\|=\left\|x^{*}\right\|$ $=1$, for $T$ is an isometry on $L$. Let $y^{*}$ be any Hahn-Banach extension of $v^{*}$ to $F . y^{*} \circ T$ is then a Hahn-Banach extension of $x^{*} \mid L$ and therefore $x^{*}=y^{*} \circ T$. For any $x \in E$ we have $\|T x\| \geqslant\left|\left\langle T x, y^{*}\right\rangle\right|=\left|\left\langle x, y^{*} \circ T\right\rangle\right|$ $=\left|\left\langle x, x^{*}\right\rangle\right|$ and so $\|T x\| \geqslant \sup \left\{\left|\left\langle x, x^{*}\right\rangle\right|: x^{*} \in S^{*}(E, L)\right\}$. If $x$ is orthogonal to $E^{*}(L)^{\perp}$, then the above remark with $D=E^{*}(L)^{\perp}$ shows that

$$
\begin{aligned}
\|x\| & =\sup \left\{\left|\left\langle x, x^{*}\right\rangle\right|: x^{*} \in E^{*}(L),\left\|x^{*}\right\|=1\right\} \\
& =\sup \left\{\left|\left\langle x, x^{*}\right\rangle\right|: x^{*} \in S^{*}(E, L)\right\} .
\end{aligned}
$$

Hence $\|T x\| \geqslant\|x\|$, which, together with $\|T\|=1$, implies $\|T x\|=\|x\|$. If $x \in L$, then $\|T x\|=\|x\|$, for $T$ is an isometry on $L$.

Corollary 2. If $E^{*}(L)=E^{*}$, then $T$ is an isometry on $E$.

After Phelps [7] we say that a subspace $L$ of $E$ has property $U$ in $E$ if every bounded linear functional on $L$ has a unique Hahn-Banach extension to $E$, i.e., $S^{*}(E, L)$ coincides with the set of $x^{*} \in E^{*}$ for which $\left\|x^{*}\right\|=\left\|x^{*} \mid L\right\|$ $=1$.

COROLlARY 3. If $L$ has property $U$ in $E$ and if $E \subseteq L^{* *}$, then $T$ is an isometry on $E$.

We next consider another aspect of Rosenthal's result. For a subset $\mathcal{L}$ of $L^{*}$ let $H(E, \mathcal{L})$ be the set of $x \in E$ such that all Hahn-Banach extensions from $L$ to $E$ of any element in $\mathcal{L}$ coincide at $x . H(E, \mathcal{L})$ is the largest subspace of $E$ containing $L$ to which every element in $\mathcal{L}$ has a unique Hahn-Banach extension.

THEOREM 4. Let $\mathcal{E} \subseteq L^{*}$ be such that the set of $y^{*} \in F^{*}$ for which $\left(y^{*} \circ T\right) \mid L \in \mathcal{L}$ and $\left\|y^{*}\right\|=\left\|y^{*} \mid M\right\|$ separates points of $F$. If $T_{1}$ is a linear operator from $E$ into $F$ such that $\left\|T_{1}\right\|=1$ and $T_{1}|L=T| L$, then $T_{1}(x)=T(x)$ for any $x \in H(E, \mathcal{L})$.

Proof. Let $x \in H(E, \mathcal{L})$ and suppose, on the contrary, that $T_{1}(x) \neq T(x)$. By the hypothesis on $F$ there exists a $y^{*} \in F^{*}$ such that $\left(y^{*} \circ T\right) \mid L \in \mathcal{L}$. $\left\|y^{*}\right\|=\left\|y^{*} \mid M\right\|$ and $\left\langle T_{1}(x), y^{*}\right\rangle \neq\left\langle T(x), y^{*}\right\rangle$. Since $y^{*} \circ T$ and $y^{*} \circ T_{1}$ are Hahn-Banach extensions to $E$ of the functional $\left(y^{*} \circ T\right) \mid L \in \mathcal{L}$, they coincide at the point $x$, which contradicts the choice of $y^{*}$.

Corollary 5. If $E=H\left(E, L^{*}\right)$ and the set of $y^{*} \in F^{*}$ with $\left\|y^{*}\right\|$ $=\left\|y^{*} \mid M\right\|$ separates points of $F$, then $T$ is uniquely determined by its values on $L$. 
The condition on $F$ given in this corollary is satisfied if $F \subseteq M^{* *}$, because $M^{*}$ separates points of $M^{* *}$ and the norm of each $v^{*} \in M^{*}$ is the same as the norm of $v^{*}$ as a linear functional on $M^{* *}$ (and so as a linear functional on $F$ ).

CoRollary 6. Suppose that (i) $L$ has property $U$ in $E$, (ii) $E^{*}(L)=E^{*}$, (iii) the set of $y^{*} \in F^{*}$ with $\left\|y^{*}\right\|=\left\|y^{*} \mid M\right\|$ separates points of $F$. Then $T$ is an isometry and is determined by its values on $L$.

EXAMPLE 1. Let $\Sigma$ be a compact Hausdorff space and $\Sigma_{0}$ a closed subset of $\Sigma$. If $C(\Sigma)$ denotes the Banach space of continuous real (or complex) functions on $\Sigma$ with the supremum norm, then the subspace $C\left(\Sigma \mid \Sigma_{0}\right)$ $=\left\{f \in C(\Sigma): f \mid \Sigma_{0}=0\right\}$ has property $U$ in $C(\Sigma)$ (cf. Phelps [7]). If $\Sigma \backslash \Sigma_{0}$ is dense in $\Sigma$, then it is easy to see that $C(\Sigma)$ is contained in the second dual of $C\left(\Sigma \mid \Sigma_{0}\right)$. If $\beta N$ denotes the Cech compactification of the set $N$ of positive integers, then $(m)$ is isometrically isomorphic with $C(\beta N)$ and $\left(c_{0}\right)$ with $C(\beta N \mid \beta N \backslash N)$. Hence $\left(c_{0}\right)$ has property $U$ in $(m)$ and moreover $(m)$ $=\left(c_{0}\right)^{* *}$. So Rosenthal's result cited above follows from Corollaries 2 and 6 . Concerning property $U$, we know that every two-sided ideal $J$ of a $C^{*}$-algebra $A$ has this property in $A$, of which the pair $\left\{\left(c_{0}\right),(m)\right\}$ is a special case. A much stronger result is contained in Dixmier [2, Proposition 2.11.7]. It is also seen that every hereditary subalgebra of a $C^{*}$-algebra $A$ has property $U$ in $A$.

EXAMPLE 2. We need neither the property $U$ for $L$ nor the fact like $E \subseteq L^{* *}$ in order to assert that $T$ is an isometry. This is illustrated by the well-known example in Korovkin's theory of approximation. Let $E=C([0,1])$ be the space of all continuous real (or complex) functions on the closed interval $[0$, 1] and $L$ the subspace of $E$ spanned by three functions; $1, t$ and $t^{2}$. Although $L$ does not have property $U$ in $E$, every evaluation functional $\varepsilon_{a}: f \rightarrow f(a)$ with $a \in[0,1]$ on the space $L$ has a unique Hahn-Banach extension, namely $\varepsilon_{a}$, to $E$. Since the set $\mathcal{E}=\left\{\varepsilon_{a}: a \in[0,1]\right\}$ coincides with the set of extreme points of the unit ball of $E^{*}$ and is contained in $S^{*}(E, L)$, we see that $E^{*}(L)=E^{*}$. So Corollary 2 applies to this case. On the other hand, we have $E$ $=H(E, \mathcal{E} \mid L)$. If the set of $y^{*} \in F^{*}$ for which $y^{*} \circ T=\varepsilon_{a}$ on $L$ for some $a \in[0,1]$ separates points of $F$, then $T$ is uniquely determined by its values on $L$. In particular, if $T$ is a linear operator from $C([0,1])$ into itself with $\|T\|=1$ and if $T(1)=1, T(t)=t$ and $T\left(t^{2}\right)=t^{2}$, then $T$ is the identity operator on $C([0,1])$.

EXAmple 3. Let $E=(m)$. Then the subspace $\left(c_{0}\right)$ has a proper closed subspace $L$ for which $E^{*}(L)=E^{*}$. Let $\left\{a_{n}: n=1,2, \ldots\right\}$ be a sequence such that, for each $k,\left|a_{k}\right|<\sum_{n \neq k}\left|a_{n}\right|<\infty$ and $L$ the set of all sequences $x=\left(x_{1}, x_{2}, \ldots\right)$ in $\left(c_{0}\right)$ with $\sum_{n=1}^{\infty} a_{n} x_{n}=0$. It is clear that $L$ is a closed subspace of $\left(c_{0}\right)$. It is also easy to see that each evaluation functional $\varepsilon_{n}: x \rightarrow x_{n}$ has a unique Hahn-Banach extension to $\left(c_{0}\right)$ and consequently to $E$. It follows that $E^{*}(L)=E^{*}$ and Corollary 2 applies to this $L$. $L$ does not contain any nontrivial ideal of $\left(c_{0}\right)$ (or $(m)$ ) and thus does not have property 
$U$ in $\left(c_{0}\right)($ or $(m))$. This last fact has been observed by $\mathrm{S}$. Takahasi in a more general situation. It is seen also that $L^{* *}$ is strictly smaller than $(m)$.

Here we include the following very slight modification of Kurtz's theorem [6, Theorem 3].

Proposition 7. Let $\mathscr{F}$ be a subset of the unit sphere $\left\{y^{*} \in F^{*}:\left\|y^{*}\right\|=1\right\}$ in $F^{*}$ such that $y^{*} \circ T \in S^{*}(E, L)$ for any $y^{*} \in \mathscr{F}$. Let $\left\{T_{\lambda}\right\}$ be a net of linear operators from $E$ into $F$ with $\left\|T_{\lambda}\right\| \leqslant 1$ such that $\left\|T_{\lambda} x-T x\right\| \rightarrow 0$ for all $x \in L$. Then, for each $x \in E,\left\langle T_{\lambda} x, y^{*}\right\rangle \rightarrow\left\langle T x, y^{*}\right\rangle$ uniformly on all $\sigma\left(F^{*}, F\right)$ compact subsets of $\mathscr{F}$. In particular, if $\mathscr{F}$ contains the $\sigma\left(F^{*}, F\right)$-closure of the extreme points of the unit sphere in $F^{*}$, then $\left\|T_{\lambda} x-T x\right\| \rightarrow 0$ for each $x \in E$.

2. Simultaneous extensions. Let $\Sigma$ be a completely regular Hausdorff space and $C(\Sigma)$ the Banach space of all bounded continuous real (or complex) functions on $\Sigma$ with the supremum norm. Let $\Omega$ be a subspace of $\Sigma$. If $X$ and $Y$ are subspaces of $C(\Omega)$ and $C(\Sigma)$, respectively, then a simultaneous extension is, by definition, a linear bounded operator $T$ from $X$ into $Y$ such that $T(f) \mid \Omega=f$ for all $f \in X$. The result of Phelps cited above implies the following, which extends Banilower [1, Corollary 1.3].

Proposition 8. Let $\Sigma$ be a completely regular Hausdorff space and $\Omega$ a locally compact subspace of $\Sigma$. Let $C_{0}(\Omega)$ be the subspace of $C(\Omega)$ consisting of elements $f$ which vanish at infinity. If $T$ is a linear operator from $C(\Omega)$ into $C(\Sigma)$ with $\|T\|=1$ and $T \mid C_{0}(\Omega)$ is a simultaneous extension, then $T$ is a simultaneous extension.

Proof. Let $R: C(\Sigma) \rightarrow C(\Omega)$ be the restriction operator. Then $R \circ T$ maps $C(\Omega)$ into $C(\Omega)$. Our assumption says that $\|R \circ T\|=1$ and $R \circ T$ induces the identity operator on $C_{0}(\Omega)$. By Corollary $5, R \circ T$ is the identity operator, as was to be proved.

The examples in $\$ 1$ furnish other kinds of simultaneous extensions. For instance we have

PROPOSITION 9. Let $\varphi$ be a homeomorphism of $[0,1]$ into a completely regular Hausdorff space $\Sigma$ and $T$ a linear operator from $C([0,1])$ into $C(\Sigma)$ with $\|T\| \leqslant 1$. If $T$ induces a simultaneous extension on the space spanned by $1, t, t^{2}$ in the sense that $(T f)(\varphi(t))=f(t)$ for $f=1, t, t^{2}$, then the same equality holds for any $f \in C([0,1])$.

Finally we extend [1, Proposition 1.4 and Theorem 1.5].

LEMMA 10. Let $\Sigma$ be a compact Hausdorff space and $\Omega$ a completely regular Hausdorff space which is extremally disconnected in the sense that the closure of every open set in $\Omega$ is open. If $V$ is a linear isometric operator from $C(\Omega)$ into $C(\Sigma)$, then there exists a homeomorphism $\pi$ from $\Omega$ into $\Sigma$ such that, for any $f \in C(\Omega)$ and any $x \in \Omega$,

$$
|(V(1))(\pi(x))|=1
$$




$$
(V(f))(\pi(x))=f(x) \cdot(V(1))(\pi(x)) .
$$

Proof. Since $V$ is an isometry, the transposed mapping $V^{*}$ of $V$ maps the closed unit ball $B^{*}(\Sigma)$ of the dual $C(\Sigma)^{*}$ onto the closed unit ball $B^{*}(\Omega)$ of $C(\Omega)^{*}$. For each $x \in \Omega$ let $\varepsilon_{x}$ be the evaluation functional $f \rightarrow f(x)$ on $C(\Omega)$. Since $C(\Omega)$ and $C(\beta \Omega)$ are isometrically isomorphic under the canonical mapping, we see that $\varepsilon_{x}$ is an extreme point of the ball $B^{*}(\Omega)$. So the set $\left(V^{*}\right)^{-1}\left(\varepsilon_{x}\right) \cap B^{*}(\Sigma)\left(=K(x)\right.$, say) is a support of $B^{*}(\Sigma)$, which is convex and weakly* compact. Let $\mu$ be an extreme point of $K(x)$, which exists by the Krein-Milman theorem. Since $K(x)$ is a support of $B^{*}(\Sigma), \mu$ is an extreme point of $B^{*}(\Sigma)$, so that there exist a point $y \in \Sigma$ and a number $\alpha,|\alpha|=1$, satisfying $\mu=\alpha^{-1} \varepsilon_{y}$ in view of [4, Lemma 7]. Thus, for each $x \in \Omega$, there exist a point $y \in \Sigma$ and a number $\alpha$ with $|\alpha|=1$ such that

$$
\begin{aligned}
(V(f))(y) & =\left\langle V(f), \varepsilon_{y}\right\rangle=\left\langle f, V^{*}\left(\varepsilon_{y}\right)\right\rangle=\left\langle f, \alpha V^{*}(\mu)\right\rangle \\
& =\left\langle f, \alpha \varepsilon_{x}\right\rangle=\alpha f(x)
\end{aligned}
$$

for all $f \in C(\Omega)$. We define, for each $x \in \Omega, \psi(x)$ to be the set of all $y \in \Sigma$ such that there exists a number $\alpha(y)$ with $|\alpha(y)|=1$ satisfying $(V(f))(y)$ $=\alpha(y) f(y)$ for all $f \in C(\Omega)$. It is easy to see that $\psi(x)$ is closed for every $x \in \Omega$ and the mapping $\psi: x \rightarrow \psi(x)$ is an upper semicontinuous mapping from $\Omega$ into the family of nonvoid compact subsets of $\Sigma$, i.e., $\{x \in \Omega$ : $\left.\psi(x) \subseteq \Sigma^{\prime}\right\}$ is open in $\Omega$ if $\Sigma^{\prime}$ is open in $\Sigma$. By use of a continuous selection theorem [5, Theorem 1.1] we can find a continuous mapping $\pi$ from $\Omega$ into $\Sigma$ such that $\pi(x) \in \psi(x)$ for any $x \in \Omega$. Since the subsets $\psi(x)$ are mutually disjoint, $\pi$ is one-to-one. We have shown that, for any $f \in C(\Omega)$ and any $x \in \Omega,(V(f))(\pi(x))=\alpha(\pi(x)) f(x)$. If $f \equiv 1$, then we have $(V(1))(\pi(x))$ $=\alpha(\pi(x))$ for any $x \in \Omega$. Since $|\alpha(y)|=1$, we have proved the statements (1) and (2). Finally let $\left\{x_{\lambda}\right\}$ be a net in $\Omega, x \in \Omega$ and suppose that $\pi\left(x_{\lambda}\right)$ tend to $\pi(x)$. Then (2) implies that $f\left(x_{\lambda}\right)$ tend to $f(x)$ for any $f \in C(\Omega)$. Since $\Omega$ is completely regular, we see that $x_{\lambda} \rightarrow x$ in $\Omega$. Hence $\pi$ is a homeomorphism.

THEOREM 11. Let $\Sigma$ be a compact Hausdorff space and $\Omega$ an extremally disconnected, completely regular Hausdorff space. Then $C(\Sigma)$ contains a subspace isometrically isomorphic to $C(\Omega)$ if and only if there exists a subspace $\Sigma_{0}$ of $\Sigma$ such that $\Sigma_{0}$ is homeomorphic with $\Omega$ and there is a simultaneous extension $T$ from $C\left(\Sigma_{0}\right)$ into $C(\Sigma)$ with norm one.

Proof. We have only to prove the necessity of the theorem. Let $V$ be a linear isometric mapping from $C(\Omega)$ into $C(\Sigma)$. Then there exists a homeomorphism $\pi$ from $\Omega$ into $\Sigma$ satisfying the condition of the preceding lemma. We set $\Sigma_{0}=\pi(\Omega)$. Define $Q: C\left(\Sigma_{0}\right) \rightarrow C(\Omega)$ by setting

$$
(Q(g))(x)=g(\pi(x)) /(V(1))(\pi(x)) .
$$

We see that $Q$ is a linear isometry from $C\left(\Sigma_{0}\right)$ onto $C(\Omega)$ and therefore that 
$T=V \circ Q$ is an isometry from $C\left(\Sigma_{0}\right)$ into $C(\Sigma)$, which is easily seen to be a simultaneous extension from $C\left(\Sigma_{0}\right)$ into $C(\Sigma)$, as was to be proved.

This theorem is reduced to [1, Theorem 1.5], when $\Omega$ is the discrete space of positive integers.

\section{REFERENCES}

1. H. Banilower, Simultaneous extensions from discrete subspaces, Proc. Amer. Math. Soc. 36 (1972), 451-455. MR 47 \# 7684.

2. J. Dixmier, Les $C^{*}$-algebres et leurs représentation, Gauthier-Villars, Paris, 1964. MR 30 \# 1404.

3. N. Dunford and J. T. Schwartz, Linear operators, Part I, Interscience, New York, 1958. MR 22 \#8302.

4. M. Hasumi, The extension property of complex Banach spaces, Tôhoku Math. J. (2) 10 (1958), 135-142. MR 20 \#7209.

5. - A contimuous selection theorem for extremally disconnected spaces, Math. Ann. 179 (1969), 83-89. MR 40 \#2024.

6. L. C. Kurtz, Unique Hahn-Banach extensions and Korovkin's theorem, Proc. Amer. Math. Soc. 47 (1975), 413-416.

7. R. R. Phelps, Uniqueness of Hahn-Banach extensions and unique best approximation, Trans. Amer. Math. Soc. 95 (1960), 238-255. MR 22 \#3964.

Departmant of Mathematics, Ibaraki UniverstTy, Mito, Ibaraki, Japan

Current address: Institut Mittag-Leffler, Auravägen 17, S-18262 Djursholm, Sweden 